\title{
Training the next generation of biomedical investigators in glycosciences
}

\author{
Peter Agre, ${ }^{1}$ Carolyn Bertozzi, ${ }^{2}$ Mina Bissell, ${ }^{3}$ Kevin P. Campbell, ${ }^{4}$ Richard D. Cummings, ${ }^{5}$ Umesh R. Desai, ${ }^{6}$ Mary Estes, \\ Terence Flotte, ${ }^{8}$ Guy Fogleman, ${ }^{9}$ Fred Gage, ${ }^{10}$ David Ginsburg, ${ }^{11}$ Jeffrey I. Gordon, ${ }^{12}$ Gerald Hart, ${ }^{13}$ Vincent Hascall, ${ }^{14}$ \\ Laura Kiessling, ${ }^{15}$ Stuart Kornfeld, ${ }^{16}$ John Lowe, ${ }^{17}$ John Magnani, ${ }^{18}$ Lara K. Mahal, ${ }^{19}$ Ruslan Medzhitov, ${ }^{20}$ Richard J. Roberts, ${ }^{21}$ \\ Robert Sackstein, ${ }^{22}$ Rita Sarkar, ${ }^{23}$ Ronald Schnaar, ${ }^{24}$ Nancy Schwartz, ${ }^{25}$ Ajit Varki, ${ }^{26}$ David Walt, ${ }^{27}$ and Irving Weissman ${ }^{28}$ \\ 'Department of Molecular Microbiology and Immunology, Johns Hopkins Bloomberg School of Public Health, Johns Hopkins University, Baltimore, Maryland, USA. ${ }^{2}$ Department of Chemistry, \\ Stanford University, Stanford, California, USA. ' Lawrence Berkeley National Laboratory, Division of Biological Systems and Engineering, Berkeley, California, USA. ${ }^{4}$ Howard Hughes Medical Institute, \\ Departments of Molecular Physiology and Biophysics, Neurology, and Internal Medicine, Carver College of Medicine, University of lowa, lowa City, lowa, USA. ${ }^{5}$ Department of Surgery, Harvard Medical \\ School, Beth Israel Deaconess Medical Center, Boston, Massachusetts, USA. ${ }^{6}$ Department of Medicinal Chemistry and Institute for Structural Biology, Drug Discovery and Development, Virginia \\ Commonwealth University, Richmond, Virginia, USA. 'Department of Molecular Virology and Microbiology, Baylor College of Medicine, Houston, Texas, USA. ${ }^{8}$ Department of Pediatrics, University of \\ Massachusetts Medical School, Worcester, Massachusetts, USA. ${ }^{9}$ Federation of American Societies for Experimental Biology (FASEB), Bethesda, Maryland, USA. ${ }^{10}$ Laboratory of Genetics LOG-G, \\ The Salk Institute for Biological Studies, La Jolla, California, USA. "Department of Internal Medicine, Department of Human Genetics, and Department of Pediatrics, University of Michigan, Ann Arbor, \\ Michigan, USA. ${ }^{12}$ Center for Genome Sciences and Systems Biology, Washington University, St. Louis, Missouri, USA. ${ }^{13}$ Department of Biological Chemistry, Johns Hopkins University, Baltimore, \\ Maryland, USA. ${ }^{14}$ Department of Bioengineering, Cleveland Clinic Foundation, Cleveland, Ohio, USA. ${ }^{15}$ Department of Chemistry, University of Wisconsin-Madison, Madison, Wisconsin, USA. \\ ${ }^{16}$ Department of Medicine, Washington University, St. Louis, Missouri, USA. ${ }^{17}$ Department of Research Pathology, Genentech, South San Francisco, California, USA. ${ }^{18} \mathrm{ClycoMimetics,} \mathrm{Rockville,} \mathrm{Maryland,} \mathrm{USA.}$ \\ ${ }^{19}$ Biomedical Chemistry Institute, Department of Chemistry, New York University, New York, New York, USA. ${ }^{20}$ Department of Immunobiology, Yale University, New Haven, Connecticut, USA. \\ ${ }^{21}$ New England BioLabs Inc., Ipswich, Massachusetts, USA. ${ }^{22}$ Departments of Dermatology and Medicine, Brigham and Women's Hospital, Harvard Medical School, Boston, Massachusetts, USA. ${ }^{23}$ Division \\ of Blood Diseases and Resources, National Heart, Lung, and Blood Institute (NHLBI), Bethesda, Maryland, USA. ${ }^{24}$ Departments of Pharmacology and Neuroscience, Johns Hopkins University, Baltimore, \\ Maryland, USA. ${ }^{25}$ Departments of Pediatrics and Biochemistry and Molecular Biology, University of Chicago, Chicago, Illinois, USA. ${ }^{26}$ Departments of Medicine and Cellular and Molecular Medicine, UCSD, \\ San Diego, California, USA. ${ }^{27}$ Department of Chemistry, Tufts Institute for Innovation, Tufts University, Medford, Massachusetts, USA. ${ }^{28}$ Institute for Stem Cell Biology and Regenerative Medicine, \\ Ludwig Center for Cancer Stem Cell Research, Stanford University, Stanford, California, USA.
}

\begin{abstract}
This position statement originated from a working group meeting convened on April 15, 2015, by the NHLBI and incorporates follow-up contributions by the participants as well as other thought leaders subsequently consulted, who together represent research fields relevant to all branches of the NIH. The group was deliberately composed not only of individuals with a current research emphasis in the glycosciences, but also of many experts from other fields, who evinced a strong interest in being involved in the discussions. The original goal was to discuss the value of creating centers of excellence for training the next generation of biomedical investigators in the glycosciences. A broader theme that emerged was the urgent need to bring the glycosciences back into the mainstream of biology by integrating relevant education into the curricula of medical, graduate, and postgraduate training programs, thus generating a critical sustainable workforce that can advance the much-needed translation of glycosciences into a more complete understanding of biology and the enhanced practice of medicine.
\end{abstract}

\section{Background and current status}

Every living cell in nature that has emerged from more than 3 billion years of biological evolution is composed of nucleic acids, proteins, lipids, metabolites, and glycans, and each of these com-

Authorship note: R. Sarkar is the NHLBI program representative. A. Varki and R. Schnaar co-chaired the working group.

Conflict of interest: J.B. Lowe is employed by and holds stock in Roche. T. Flotte has worked as a consultant at Dimension Therapeutics. D. Walt is a member of the Boards of Directors of Illumina, Cerulean, Quanterix, Exicure, and Ultivue Inc. J.L. Magnani is employed by and holds stock in GlycoMimetics. I. Weissman has worked as a consultant and holds stock in Stem Cells Inc. and Forty Seven Inc. J.I. Gordon is a cofounder of Matatu Inc., a company characterizing the role of diet-by-microbiota interactions in animal health. R.J. Roberts is employed by New England BioLabs Inc., which sells glycobiology reagents in its product catalog.

Reference information: J Clin Invest. 2016;126(2):405-408. doi:10.1172/JCI85905. ponents remains essential to all life forms. Until the 1970s, the study of glycans was thus an integral part of the exploration of all biological systems, generating major advances in fields as diverse as hematology, microbiology, metabolism, and material sciences. With the rapid advent of powerful insights and tools, the study of nucleic acids and proteins came to dominate the molecular biology revolution of subsequent decades. While similar revolutions in the biological exploration of lipids and metabolites have now followed, the study of glycans has been left far behind. The reasons for this omission are many, but can be broadly ascribed to the far greater complexity and diversity of this class of molecules and the related lack of comparable tools for their exploration. In analogy to the situation in cosmology, the "standard model" for approaching biological systems now largely excludes glycans, which have effectively become the "dark matter" of the biological universe: criti- 
cal for a full understanding of biology and yet routinely ignored. While a small number of scientists persisted in the in-depth study of glycans (resulting in a specialized field called glycobiology), an entire generation of mainstream biologists was trained with little knowledge of the structure, analysis, or functions of glycans or even of the terminology required for discussing them. This anomaly in the history of the biological sciences has since trickled down to all levels of education, resulting in lost opportunities for a more complete understanding of the biology and pathology of living systems, which would also greatly benefit therapeutic development and medical practice. Very few scientists are thus now trained in understanding glycosciences, and studies of glycans have also lost prominent attention from major journals and grant review groups or recognition by honors and awards.

Recognizing this stark deficiency, the NIH, the National Science Foundation (NSF), the Department of Energy (DOE), the FDA, and the Howard Hughes Medical Institute (HHMI) commissioned an enquiry into the situation by the National Research Council (NRC) of the National Academies (1). The resulting 2012 report, entitled Transforming Glycoscience: A Roadmap for the Future, noted the following:

Glycoscience is a highly interdisciplinary field that aims to better understand the structures and functions of glycans and how they can be used. It is a global field with a dedicated community of researchers in the United States and abroad. Glycoscientists do not have a single training/education background. They come from various fields, including physiology and developmental biology, where glycans are involved in processes such as cell movement and tissue development. They are in medicine, where glycans are involved in the development and progression of chronic and infectious diseases. In microbiology, glycans are key players in interactions among and between microbes and host cells. Glycoscientists are chemists developing new synthetic and analytical methods for glycans, and biochemists working to understand glycan synthesis and metabolism. In materials science, glycans can be used as polymeric materials having a wide range of properties. In computational science and informatics, modeling studies and the effective analysis of large amounts of experimental data are also necessary to better understanding glycans.

In the area of human health, the NRC report also noted that (a) glycans are directly involved in the pathophysiology of every major disease; (b) additional knowledge from glycosciences will be needed to realize the goals of personalized medicine and to take advantage of the substantial investments in human genome and proteome research; and (c) glycans are increasingly important in pharmaceutical development.

Among the resulting roadmap goals in the NRC report were specific comments and recommendations regarding enhanced training in the glycosciences.

The committee notes that there is widespread lack of understanding and appreciation of glycoscience in the scientific and medical communities and among the general pub- lic. Glycans are integral components of living organisms, whether human, animal, plant, or microbe, and glycan products have applications in health, energy, and materials science. The committee concludes that integrating glycoscience into relevant disciplines in high school, undergraduate, and graduate education, and developing curricula and standardized testing for science competency would increase public as well as professional awareness.

Within 10 years, glycoscience will be integrated and taught at every level wherever it is relevant to understand the scientific content.

In response to the overall NRC report, the NIH Office of Strategic Coordination via the NIH Director's Common Fund released a series of requests for applications with the following program initiatives: (a) developing methods and technologies for synthesis of biomedically relevant carbohydrates; (b) developing accessible tools for probing and analyzing carbohydrates and their interaction partners; (c) developing data integration and analysis tools.

Notably lacking was any direct support for training in glycosciences. It was the charge of this working group to consider further the need for mechanisms to address this unmet need.

\section{Working group objectives}

The working group defined its objectives as follows: "Discuss the value and mechanisms of training the next generation of biomedical investigators in the glycosciences, with the goal of bringing glycoscience back into the mainstream of biology - by generating a critical sustainable workforce that will advance the translation of glycosciences in biology and medicine, and help integrate glycoscience education into the curricula of medical and graduate schools" (1).

\section{Definition of needs and opportunities}

There is increasing recognition of the diverse roles and uses of glycans in biology and medicine. Yet glycoscience has not gained much attention in the mainstream of biology and is viewed as a niche research area with relatively few qualified researchers. While high school, undergraduate, and graduate students learn about nucleic acids, proteins, lipids, and metabolites, their competency in understanding the chemistry and biology of glycans is now conspicuously deficient. Also, compared with the NIH investments made in understanding other major components of life, glycans have received very little attention. This chasm needs to be addressed by training a new generation of biomedical researchers for whom glycans are an integral part of their mainstream thinking.

The working group discussed the role of glycosciences in the discovery process. One theme that repeatedly arose was that the field is indeed poorly represented among researchers and lecturers at most biomedical research institutions. Despite this, some great advances in biomedical discovery in several areas, including hematology, immunology, infectious diseases, genetic diseases, cancer, and neuroscience, have been facilitated by the coincidental proximity of key investigators to colleagues who understood the glycoscience aspects of their research and also had the motivation and tools to apply this understanding to the biomedical challenges at hand. Without such serendipitous input, the discoveries 


\section{Building a workforce versed in glycoscience}

Add basic knowledge of glycoscience to national standardized tests to encourage inclusion of the discipline in teaching.

Include more key concepts of glycoscience in undergraduate and graduate level textbooks in the biomedical sciences (e.g., cell biology, biochemistry).

Encourage opportunities for hands-on training in glycoscience experimentation and discovery.

"Democratize" glycoscience for nonspecialists. Train life scientists and clinicians to be conversant in the discipline, even if they never become experts.

Encourage glycoscientists to seek opportunities to lecture outside of the field in order to disseminate knowledge, break down barriers, and motivate others to embrace glycoscience within their biomedical world view.

Integrate glycosciences in their appropriate place in all major chemical and biomedical disciplines, instead of as a field apart. Make glycobiology integral to biology and glycochemistry integral to biochemistry and organic chemistry.

Emphasize the attractive biological functional aspects of the field to motivate students and colleagues to delve more deeply into its details.

Promote existing resources (textbooks, online content) to help established faculty expand glycoscience teaching.

Leverage opportunities from successful pharmaceutical developments that have been highlighted online and in the popular press to emphasize positive outcomes arising from glycosciences.

Encourage training of those at regulatory and governmental bodies in the opportunities of glycosciences.

would have been delayed or not been made at all. Furthermore, without knowledgeable faculty in many biomedical educational institutions, there is a striking absence of lectures and seminars in the area. This creates a feed-forward self-perpetuating deficit of knowledge in the field. Glycosciences are not taught (or are barely taught) at many institutions, leading to a generation of otherwise superbly trained physicians and biomedical scientists who are nonetheless unfamiliar with language and basic knowledge in this key aspect of all biological systems. When such individuals enter the biomedical workforce, whether in academia, science administration, or the drug discovery and diagnostics industries, they are unlikely to grasp the implications of glycosciences for their own work or that of others. They are also unlikely to sufficiently emphasize glycoscience training in the curricula they develop. Generation after generation, we are now losing ground with regard to the potential of this discipline.

For those few who do make glycosciences their career, the rewards of discovery are hard fought, often against significant resistance from editorial boards, grant review committees, and biomedical investment groups who lack the basic knowledge of glycosciences necessary to understand the goals and opportunities at hand. Despite these challenges, significant advances have been translated from glycoscience discovery to the clinic. For example, many groundbreaking biologicals now in the clinic, including cytokines, such as erythropoietin and all monoclonal antibodies, are glycoproteins that are expressly dependent on their glycans for pharmacodynamic and pharmacokinetic properties, stability, and function. Important vaccines have been developed based on microbial glycans, such as pneumococcus and haemophilus polysaccharides. In addition, glycomimetic compounds developed to target glycan-binding sites on functional glycanbinding proteins are now showing promise for syndromes such as sickle cell crises, with more under development by the biotech and pharmaceutical industries.

A consensus arose on the committee that the basic and therapeutic significance of glycosciences has already been well established, with more progress on the way. But maintaining and accelerating translation in glycosciences will require a reversal of the ongoing year-over-year deficit of knowledge in this area among most biomedical scientists. One solution will be to populate major academic research centers, research institutes, government laboratories, and drug development companies with colleagues who have experience and knowledge in the glycosciences. But the pool of such individuals is currently very small. Potential resources that will help correct this situation are discussed further below. In addition, we believe several key actions are required to bring more of our trainees and colleagues on board, as detailed in Building a workforce versed in glycoscience.

\section{Developing resources for glycoscience training}

It is the consensus of the working group that bottom-up rebuilding of the glycoscience enterprise with a long-range perspective will enhance all aspects of biology and medicine. In particular, investing resources in populating the biomedical research enterprise at all levels with individuals trained in the discipline will have outsized positive effects on discovery. The presence of trained glycoscientists as researchers, faculty members, science administrators, reviewers, journal editors, biomedical investors, patent officers, and consultants will reverse the "glycoscience desert" at many institutions and levels and begin to infuse 


\section{Expanding support for glycoscience training}

Create centers of excellence for training the next generation of biomedical investigators in the glycosciences. As an example, establish new K12 and K01 mechanisms to support supervised transition of biomedical scientists late in training to independent careers with full integration of the glycosciences. It is anticipated that such awards would incentivize established experts to recruit and mentor young colleagues and to expand the presence of independent glycoscience-knowledgeable investigators across the biosciences.

Provide independent support for those with recent glycoscience-related doctorates to pursue glycoscience-related postdoctoral research collaboratively in laboratories that are not traditionally glycoscience focused. This support will encourage those with glycoscience needs to access expertise and expand the knowledge base of their laboratories in this discipline.

Create a collaborative research award for a laboratory in need of glycoscience expertise to work with an established glycoscience laboratory. This could perhaps take the form of supplements to existing R01 grants of the two labs in question. This mechanism should be considered by all NIH institutes whose portfolios are relevant to glycosciences.

Support fellowships for undergraduate and medical students to perform summer projects in glycoscience laboratories.

Encourage NIH institutes to include glycoscience training in the form of special-emphasis training grants.

Expand upon the few existing immersive glycoscience training courses to bring investigators who are not in the field up to speed in basic knowledge of glycosciences. A Gordon Conference or Wood's Hole/Cold Spring Harbor-style week-long or multiweek format, already successful in advanced glycoscience courses in Europe, could be a model. Multidisciplinary meetings or workshops emphasizing the roles of glycans in fields such as neuroscience, cancer, immunity, infectious diseases, blood and vascular systems, etc. would also be of value.

Encourage established glycoscientists to get more involved in basic undergraduate and graduate courses, ranging from organic chemistry to structural biology, physiology, and molecular and cellular biology.

knowledge about glycans into teaching, research, and the knowledge base of physicians and scientists.

As demonstrated by the successes of the current NHLBIsponsored Programs of Excellence in Glycoscience mechanism, a quick way to achieve this goal in the short term is to develop programs of supervised research training to bring more young investigators at the postdoctoral and starting faculty level into the field. This type of intensive immersion training of those late in their training careers has been shown to have a significant positive outcome in keeping trainees in the field when they embark on independent careers. We propose that specific steps can be taken to address this gap for young investigators, as detailed in Expanding support for glycoscience training.

\section{Concluding statement}

Glycans are ubiquitous in all living cells and organisms, where they serve essential functions, ranging from acting as structural components to regulating physiological and pathological processes. Evidence clearly indicates that glycans represent a largely untapped resource for biological discovery as well as unanticipated therapeutic opportunities. However, among the major classes of biomolecules, glycans have generally received the least attention, in part due to a self-perpetuating underappreciation of their biologic impact, their inherent structural complexity, and the lack of tools to synthesize and analyze them. This is changing, with new capabilities and new resources focused on enhancing research tools in glycosciences. While such investments are very worthwhile, they will fall short of their promise unless the stark deficit in training and knowledge in the field is vigorously addressed. The committee feels that using a bottom-up approach of emphasizing training and incentivizing an increased number of young investigators will begin to reverse the self-perpetuating underutilization of glycosciences in biomedical sciences, resulting in outsized benefits towards enhancing knowledge and tools in the discipline and thus more effectively meeting the mandates of the $\mathrm{NIH}$ in particular and the biomedical research enterprise in general.

Address correspondence to: Ajit Varki, Departments of Medicine and of Cellular and Molecular Medicine, BRF2, Room 4126, 9500 Gilman Drive, MC 0687, La Jolla, California 92093-0687, USA. Phone: 858.534.2214; E-mail: a1varki@ucsd.edu. Or to: Ronald L. Schnaar, Departments of Pharmacology and Neuroscience, Johns Hopkins University School of Medicine, 725 N. Wolfe St., 318 Wood Basic Science Bldg., Baltimore, Maryland 21205, USA. Phone: 410.955.8392; E-mail: schnaar@jhu.edu.

1. National Research Council. Transforming Glycoscience: A Roadmap for the Future. Washington, DC, USA: The National Academies Press; 2012. 